\title{
A genome-wide association study identifies a susceptibility locus for refractive errors and myopia at $15 q 14$
}

Abbas M. Solouki ${ }^{1,2 \#}$, Virginie J.M. Verhoeven ${ }^{1,2 \#}$, Cornelia M. van Duijn ${ }^{2}$, Annemieke J.M.H. Verkerk ${ }^{3}$, M. Kamran Ikram ${ }^{1,2,4}$, Pirro G. Hysi ${ }^{5}$, Dominiek D.G. Despriet ${ }^{1,2}$, Leonieke M. van Koolwijk ${ }^{1,6}$, Lintje Ho ${ }^{1,2}$, Wishal Ramdas ${ }^{1,2}$, Monika Czudowska ${ }^{1,2}$, Robert W.A.M. Kuijpers ${ }^{1}$, Najaf Amin ${ }^{2}$, Maksim Struchalin ${ }^{2}$, Yurii S. Aulchenko ${ }^{2}$, Gabriel van Rij ${ }^{1}$, Frans C.C. Riemslag ${ }^{7}$, Terri L. Young ${ }^{8}$, David A. Mackey ${ }^{9}$, Timothy D. Spector ${ }^{5}$, Theo G.M.F. Gorgels $^{10}$, J.J.M. Willemse-Assink ${ }^{11}$, Aaron Isaacs ${ }^{2}$, R. Kramer ${ }^{12}$, Sigrid M.A. Swagemakers $^{3,13,14}$, Arthur A.B. Bergen ${ }^{10,15,16}$, Andy A.L.J. van Oosterhout ${ }^{2,17}$, Ben A. Oostra $^{17}$, Fernando Rivadeneira ${ }^{2,18}$, Andre G. Uitterlinden ${ }^{2,18}$, Albert Hofman ${ }^{2}$, Paulus T.V.M. de Jong ${ }^{2,10}$, Christopher J. Hammond ${ }^{5}$, Johannes R.Vingerling ${ }^{1,2}$, Caroline C.W. Klaver ${ }^{1,2}$

${ }^{1}$ Department of Ophthalmology, Erasmus Medical Center, Rotterdam, the Netherlands ${ }^{2}$ Department of Epidemiology, Erasmus Medical Center, Rotterdam, the Netherlands ${ }^{3}$ Department of Bioinformatics, Erasmus Medical Center, Rotterdam, the Netherlands ${ }^{4}$ Department of Neurology, Erasmus Medical Center, Rotterdam, the Netherlands

${ }^{5}$ Department of Twin Research and Genetic Epidemiology, King's College London School of Medicine, London, United Kingdom

${ }^{6}$ The Rotterdam Eye Hospital, Rotterdam, the Netherlands

${ }^{7}$ Bartiméus, Institute for the Visually Impaired, Zeist, the Netherlands

${ }^{8}$ Center for Human Genetics, Duke University, Durham, North Carolina, United States of America

${ }^{9}$ Lions Eye Institute, University of Western Australia, Centre for Ophthalmology and Visual Science, Perth, Australia

${ }^{10}$ Department of Clinical and Molecular Ophthalmogenetics, Netherlands Institute of Neurosciences (NIN), An Institute of the Royal Netherlands Academy of Arts and Sciences (KNAW), Amsterdam, the Netherlands

${ }^{11}$ Department of Ophthalmology, Amphia Hospital, Breda, the Netherlands

${ }^{12}$ Department of Ophthalmology, Fransiscus Hospital, Roosendaal, the Netherlands

${ }^{13}$ Department of Genetics, Erasmus Medical Center, Rotterdam, the Netherlands

${ }^{14}$ The Cancer Genomics Center, the Netherlands

${ }^{15}$ Department of Clinical Genetics, Academic Medical Center, Amsterdam, the Netherlands

${ }^{16}$ Department of Ophthalmology, Academic Medical Center, Amsterdam, the Netherlands

${ }^{17}$ Department of Clinical Genetics, Erasmus Medical Center, Rotterdam, the Netherlands

${ }^{18}$ Department of Internal Medicine and Clinical Chemistry, Erasmus Medical Center,

Rotterdam, the Netherlands

\#These authors contributed equally to this work.

Correspondence should be addressed to: Dr. C.C.W. Klaver (C.C.W.K.), Dept. of Ophthalmology, Erasmus Medical Center Rotterdam, PO Box 2040, 3000 CA Rotterdam, Telephone: (+31) 6-51934491; Fax: (+31) 10-7033692; E-mail: c.c.w.klaver@erasmusmc.nl 
Supplementary Table 1. Clinical characteristics of study participants

Abbreviations: GWAS, genome-wide association study; RS-I, Rotterdam Study I; RS-II, Rotterdam Study II; RS-III, Rotterdam Study III; ERF, Erasmus Rucphen Family Study;

TwinsUK, the Twin Cohort recruited in London; SD, standard deviation; D, diopters.

\begin{tabular}{|c|c|c|c|c|c|c|}
\hline & & RS-I & RS-II & RS-III & ERF & TwinsUK \\
\hline \multicolumn{2}{|l|}{ Number of individuals in GWAS } & 5328 & 2008 & 1970 & 2032 & 4270 \\
\hline \multicolumn{2}{|l|}{ Percentage women (\%) } & 58.7 & 54.1 & 56.1 & 56.9 & 92.6 \\
\hline \multicolumn{2}{|l|}{ Mean age in years (SD) } & $68.5(8.6)$ & $64.2(7.4)$ & $56.2(5.8)$ & 48.5(14.3) & $55.0(12.0)$ \\
\hline \multicolumn{2}{|c|}{ Refractive error, spherical equivalent mean (SD) } & $+0.86(2.45)$ & $+0.48(2.51)$ & $-0.35(2.62)$ & $+0.07(2.13)$ & $-0.40(2.73)$ \\
\hline \multirow{2}{*}{\multicolumn{7}{|c|}{ Spherical equivalent categories: $\mathrm{n}(\%)$}} \\
\hline - High myopia & $(<=-6 \mathrm{D})$ & $91(1.7)$ & & & & \\
\hline - Moderate myopia & $(-6 \mathrm{D}:-3 \mathrm{D})$ & $271(5.1)$ & $148(7.4)$ & $244(12.4)$ & $102(5.0)$ & $410(9.6)$ \\
\hline - Low myopia & $(-3 \mathrm{D}:-1.5 \mathrm{D})$ & $276(5.2)$ & $141(7.0)$ & $199(10.1)$ & $138(6.8)$ & $420(9.8)$ \\
\hline - Emmetropia & $(-1.5 \mathrm{D}:+1.5 \mathrm{D})$ & $2538(47.6)$ & $1024(51.0)$ & $1089(55.3)$ & $1372(67.5)$ & 2532(59.3) \\
\hline - $\quad$ Low hypermetropia & $(+1.5 \mathrm{D}:+3 \mathrm{D})$ & $1403(26.3)$ & $453(22.5)$ & $254(12.9)$ & $263(12.9)$ & $502(11.8)$ \\
\hline - Moderate hypermetropia & $(+3 \mathrm{D}:+6 \mathrm{D})$ & $675(12.7)$ & $189(9.4)$ & $105(5.3)$ & $98(4.8)$ & $205(4.8)$ \\
\hline - High hypermetropia & $(>=+6 \mathrm{D})$ & $74(1.4)$ & $19(0.9)$ & $17(0.9)$ & $15(0.7)$ & \\
\hline
\end{tabular}


Supplementary Table 2. Genome-wide association (and replication) for refractive error with $31 \mathrm{SNPs}$ spread across four loci on chromosome 15q14, 14q24, 1q41, and 10p12.3 reaching $P<10^{-6}$

Abbreviations: RS-I, Rotterdam Study I; RS-II, Rotterdam Study II; RS-III, Rotterdam Study III; ERF, Erasmus Rucphen Family Study; TwinsUK, the Twin Cohort recruited in

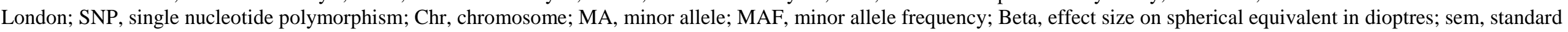
error of the mean; -, no data.

\begin{tabular}{|c|c|c|c|c|c|c|c|c|c|c|c|c|c|c|c|c|}
\hline \multirow[b]{2}{*}{ SNP } & \multirow[b]{2}{*}{ Chr } & \multirow[b]{2}{*}{ Position } & \multicolumn{4}{|c|}{$\begin{array}{l}\text { Discovery cohort: } \\
\operatorname{RS}(n=5328)\end{array}$} & \multicolumn{2}{|c|}{$\begin{array}{l}\text { Replication } \\
\text { RS-II }(n=2008)\end{array}$} & \multicolumn{2}{|c|}{ RS-III $(n=1970)$} & \multicolumn{2}{|c|}{$\operatorname{ERF}(n=2032)$} & \multicolumn{2}{|c|}{ TwinsUK $(n=4270)$} & \multicolumn{2}{|c|}{ Meta-analysis $(n=15608)$} \\
\hline & & & MA & MAF & Beta (sem) & $P$ & Beta (sem) & $P$ & Beta (sem) & $P$ & Beta (sem) & $P$ & Beta (sem) & $p$ & Beta (sem) & \\
\hline 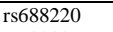 & 15 & 32786167 & A & 0.45 & $-0.27(0.05)$ & $1.76 \times 10^{-8}$ & $-0.28(0.08)$ & $3.80 \times 10^{-4}$ & $-0.22(0.08)$ & $9.27 \times 10^{-3}$ & $-0.03(0.07)$ & $6.24 \times 10^{-1}$ & $-0.15(0.07)$ & $2.60 \times 10^{-2}$ & $-0.20(0.0009)$ & $2.79 \times 10^{-11}$ \\
\hline rs580839 & 15 & 32786121 & A & 0.44 & $-0.27(0.05)$ & $1.89 \times 10^{-8}$ & $-0.27(0.08)$ & $4.96 \times 10^{-4}$ & $-0.22(0.08)$ & $7.95 \times 10^{-3}$ & $-0.03(0.07)$ & $6.34 \times 10^{-1}$ & $-0.16(0.07)$ & $1.92 \times 10^{-2}$ & $-0.20(0.0009)$ & $2.53 \times 10^{-11}$ \\
\hline rs619788 & 15 & 32782398 & A & 0.44 & $-0.27(0.05)$ & $1.92 \times 10^{-8}$ & $-0.27(0.08)$ & $4.94 \times 10^{-4}$ & $-0.22(0.08)$ & $7.72 \times 10^{-3}$ & $-0.03(0.07)$ & $6.27 \times 10^{-1}$ & $-0.16(0.07)$ & $1.85 \times 10^{-2}$ & $-0.20(0.0009)$ & $2.53 \times 10^{-11}$ \\
\hline rs4924134 & 15 & 32781857 & G & 0.44 & $-0.27(0.05)$ & $2.04 \times 10^{-8}$ & $-0.27(0.08)$ & $4.76 \times 10^{-4}$ & $-0.27(0.08)$ & $6.58 \times 10^{-3}$ & $-0.06(0.07)$ & $4.10 \times 10^{-1}$ & $-0.16(0.07)$ & $1.85 \times 10^{-2}$ & $-0.21(0.0009)$ & $1.36 \times 10^{-12}$ \\
\hline rs560766 & 15 & 32788234 & A & 0.44 & $-0.26(0.05)$ & $4.27 \times 10^{-8}$ & $-0.28(0.08)$ & $4.54 \times 10^{-4}$ & $-0.21(0.08)$ & $1.29 \times 10^{-2}$ & $-0.03(0.07)$ & $6.65 \times 10^{-1}$ & $-0.18(0.07)$ & $7.68 \times 10^{-3}$ & $-0.20(0.0009)$ & $2.49 \times 10^{-11}$ \\
\hline rs7176510 & 15 & 32786771 & $\mathrm{~T}$ & 0.45 & $-0.26(0.05)$ & $5.16 \times 10^{-8}$ & $-0.28(0.08)$ & $5.10 \times 10^{4}$ & $-0.22(0.08)$ & $9.62 \times 10^{-3}$ & $-0.02(0.07)$ & $7.51 \times 10^{-1}$ & $-0.16(0.07)$ & $1.76 \times 10^{-2}$ & $-0.20(0.0009)$ & $6.25 \times 10^{-11}$ \\
\hline rs7163001 & 15 & 32777866 & A & 0.44 & $-0.26(0.05)$ & $\begin{array}{l}5.23 \times 10^{-8} \\
576 \times 10^{-8}\end{array}$ & $-0.28(0.08)$ & $\begin{array}{l}4.08 \times 10^{-4} \\
\end{array}$ & $-0.23(0.08)$ & $5.89 \times 10^{-3}$ & $-0.07(0.07)$ & $3.01 \times 10^{-1}$ & $-0.16(0.07)$ & $1.87 \times 10^{-2}$ & $-0.21(0.0009)$ & $5.61 \times 10^{-12}$ \\
\hline $\begin{array}{l}\text { rr1110730606 } \\
\text { rs8032019 }\end{array}$ & $\begin{array}{l}15 \\
15\end{array}$ & $\begin{array}{l}327771433 \\
3278782\end{array}$ & $\begin{array}{l}\mathrm{A} \\
\mathrm{G}\end{array}$ & $\begin{array}{l}0.44 \\
0.40\end{array}$ & $\begin{array}{l}-0.26(0.05) \\
-0.26(0.05)\end{array}$ & $\begin{array}{l}5.76 \times 10^{-8} \\
609 \times 10^{-8}\end{array}$ & $\begin{array}{l}-0.28(0.08) \\
-0.02(0.08)\end{array}$ & $\begin{array}{l}4.05 \times 10^{-4} \\
5.5710^{-4}\end{array}$ & $\begin{array}{l}-0.23(0.08) \\
-0.009\end{array}$ & $5.82 \times 10^{-3}$ & $\begin{array}{l}-0.08(0.07) \\
-0.05007\end{array}$ & $\begin{array}{l}2.72 \times 10^{-1} \\
512 \times 10^{-1}\end{array}$ & $-0.16(0.07)$ & $\begin{array}{l}1.91 \times 10^{-2} \\
1.96 \times 10^{-2}\end{array}$ & $-0.21(0.0009)$ & $3.65 \times 10^{-12}$ \\
\hline rs 685352 & 15 & 32795627 & G & 0.44 & $\begin{array}{l}-0.26(0.05) \\
-0.25(0.05)\end{array}$ & $\begin{array}{l}\text {.0.09110 } \\
8.80 \times 10^{-8}\end{array}$ & $\begin{array}{l}-0.28(0.08) \\
-0.25(0.08)\end{array}$ & $\begin{array}{l}5.5 \times 10 \\
1.28 \times 10^{-3}\end{array}$ & $\begin{array}{l}-0.13(0.09) \\
-0.19(0.08)\end{array}$ & $\begin{array}{l}1.30 \times \times 10^{-2} \\
1.98 \times 0^{-2}\end{array}$ & $\begin{array}{l}-0.05(0.07) \\
-0.07(0.07)\end{array}$ & $\begin{array}{l}5.12 \times 10^{-1} \\
3.06 \times 10^{-1}\end{array}$ & $\begin{array}{l}-0.16(0.07) \\
-0.24(0.07)\end{array}$ & $\begin{array}{l}1.96 \times 10^{-2} \\
444 \times 10^{-4}\end{array}$ & $\begin{array}{l}-0.19(0.0009) \\
-0.1009\end{array}$ & $\begin{array}{l}3.7 \times 10^{-10} \\
419 \times 10^{-12}\end{array}$ \\
\hline rs 524952 & 15 & 32793178 & A & 0.47 & $0.25(0.05)$ & $1.03 \times 10^{-7}$ & $0.30(0.08)$ & $2.09 \times 10^{-4}$ & $0.19(0.08)$ & $2.56 \times 10^{-2}$ & $-0.06(0.07)$ & $4.13 \times 10^{-1}$ & $\begin{array}{l}-0.24(0.07) \\
-0.32(0.07)\end{array}$ & $\begin{array}{l}4.4 \times 10 \\
4.15 \times 10^{-6}\end{array}$ & $\begin{array}{l}-0.21(0.0009) \\
-0.23(0.0099\end{array}$ & $\begin{array}{l}4.19 \times 10^{-12} \\
3.18 \times 10^{-14}\end{array}$ \\
\hline rs634990 & 15 & 32793365 & $\mathrm{C}$ & 0.47 & $0.25(0.05)$ & $1.03 \times 10^{-7}$ & $0.30(0.08)$ & $2.15 \times 10^{-4}$ & $0.20(0.08)$ & $2.03 \times 10^{-2}$ & $-0.05(0.07)$ & $5.11 \times 10^{-1}$ & $-0.33(0.07)$ & $2.93 \times 10^{-6}$ & $-0.23(0.0009)$ & $2.21 \times 10^{-14}$ \\
\hline rs1 1073059 & 15 & 32776966 & A & 0.44 & $-0.25(0.05)$ & $1.20 \times 10^{-7}$ & $-0.28(0.08)$ & $3.96 \times 10^{-4}$ & $-0.23(0.08)$ & $5.83 \times 10^{-3}$ & $-0.08(0.07)$ & $2.72 \times 10^{-1}$ & $-0.16(0.07)$ & $1.91 \times 10^{-2}$ & $-0.20(0.0009)$ & $8.45 \times 10^{-12}$ \\
\hline rs1 1073058 & 15 & 32776918 & $\mathrm{~T}$ & 0.44 & $-0.25(0.05)$ & $1.30 \times 10^{-7}$ & $-0.28(0.08)$ & $3.93 \times 10^{-4}$ & $-0.23(0.08)$ & $5.84 \times 10^{-3}$ & $-0.08(0.07)$ & $2.7 \times 10^{-1}$ & $-0.16(0.07)$ & $1.90 \times 10^{-2}$ & $-0.20(0.0009)$ & $8.45 \times 10^{-12}$ \\
\hline rs10141617 & 14 & 69486195 & A & 0.32 & $-0.27(0.05)$ & $1.45 \times 10^{-7}$ & $-0.10(0.08)$ & $2.04 \times 10^{-1}$ & $-0.05(0.09)$ & $6.08 \times 10^{-1}$ & $-0.05(0.07)$ & $5.2 \times 10^{-1}$ & $0.10(0.07)$ & $1.46 \times 10^{-1}$ & $-0.11(0.0009)$ & $2.88 \times 10^{-4}$ \\
\hline rs12123296 & 1 & 214080199 & $\mathrm{~T}$ & 0.03 & $-0.69(0.14)$ & $3.92 \times 10^{-7}$ & $-0.09(0.23)$ & $7.07 \times 10^{-1}$ & $0.29(0.26)$ & $2.63 \times 10^{-1}$ & $-0.33(0.22)$ & $1.5 \times 10^{-1}$ & & & $-0.29(0.009)$ & $4.50 \times 10^{-3}$ \\
\hline rs12402107 & 1 & 214079366 & G & 0.03 & $-0.69(0.14)$ & $3.92 \times 10^{-7}$ & $-0.09(0.23)$ & $7.07 \times 10^{-1}$ & $0.29(0.26)$ & $2.63 \times 10^{-1}$ & $-0.33(0.22)$ & $1.5 \times 10^{-1}$ & & & $0.29(0.009)$ & $4.50 \times 10^{-3}$ \\
\hline rs12132798 & 1 & 214087159 & G & 0.03 & $-0.70(0.14)$ & $3.94 \times 10^{-7}$ & $-0.09(0.23)$ & $7.04 \times 10^{-1}$ & $0.29(0.26)$ & $2.64 \times 10^{-1}$ & $-0.33(0.23)$ & $1.5 \times 10^{-1}$ & - & - & $0.29(0.009)$ & $4.21 \times 10^{-3}$ \\
\hline rs12141208 & 1 & 214058683 & C & 0.03 & $-0.69(0.14)$ & $4.02 \times 10^{-7}$ & $-0.09(0.23)$ & $7.04 \times 10^{-1}$ & $0.29(0.26)$ & $2.55 \times 10^{-1}$ & $-0.32(0.22)$ & $1.5 \times 10^{-1}$ & - & - & $-0.29(0.009)$ & $4.61 \times 10^{-3}$ \\
\hline rs11120645 & 1 & 214057102 & $\mathrm{~T}$ & 0.03 & $-0.69(0.14)$ & $4.03 \times 10^{-7}$ & $-0.09(0.23)$ & $7.04 \times 10^{-1}$ & $0.29(0.26)$ & $2.54 \times 10^{-1}$ & $-0.32(0.22)$ & $1.5 \times 10^{-1}$ & - & - & $-0.29(0.009)$ & $4.64 \times 10^{-3}$ \\
\hline rs12137796 & 1 & 214054933 & T & 0.03 & $-0.69(0.14)$ & $\begin{array}{l}4.05 \times 10^{-7} \\
\end{array}$ & $-0.09(0.23)$ & $\begin{array}{l}7.03 \times 10^{-1} \\
66510^{-1}\end{array}$ & $0.29(0.26)$ & $2.54 \times 10^{-1}$ & $-0.32(0.22)$ & $1.5 \times 10^{-1}$ & - & - & $-0.28(0.009)$ & $4.72 \times 10^{-3}$ \\
\hline $\begin{array}{l}\text { rs1212126346 } \\
\text { rs } 12144789\end{array}$ & 1 & $\begin{array}{l}214088575 \\
214049058\end{array}$ & C & 0.03 & $\begin{array}{l}-0.70(0.14) \\
-0.69(0.14)\end{array}$ & $\begin{array}{l}4.07 \times 10^{-7} \\
421 \times 10^{-7}\end{array}$ & $\begin{array}{l}-0.10(0.24) \\
-009(0.23)\end{array}$ & $6.65 \times 10^{-1}$ & $0.29(0.26)$ & $\begin{array}{l}2.67 \times 10^{-1} \\
250 \times 0^{-1}\end{array}$ & $-0.33(0.23)$ & $1.5 \times 10^{-1}$ & & & $-0.29(0.009)$ & $4.20 \times 10^{-3}$ \\
\hline $\begin{array}{l}\mathrm{r} 1212133551 \\
\mathrm{rs} 12351\end{array}$ & 1 & 214048165 & C & $\begin{array}{l}0.03 \\
0.03\end{array}$ & $\begin{array}{l}-0.69(0.14) \\
-0.69(0.14)\end{array}$ & $\begin{array}{l}4.2 \times 1 \times 10^{-7} \\
4.24 \times 10^{-7}\end{array}$ & $\begin{array}{l}-0.09(0.23) \\
-0.09(0.23)\end{array}$ & $\begin{array}{l}7.02 \times 10^{-1} \\
7.02 \times 10^{-1}\end{array}$ & $\begin{array}{l}0.29(0.26) \\
0.29(0.26)\end{array}$ & $\begin{array}{l}2.50 \times 10^{-1} \\
2.5010^{-1}\end{array}$ & $\begin{array}{l}-0.32(0.22) \\
-0.32(0.22)\end{array}$ & $\begin{array}{l}1.5 \times 10^{-1} \\
1.5 \times 10^{-1}\end{array}$ & - & - & $\begin{array}{r}-0.28(0.0099) \\
0.28(0.009)\end{array}$ & $\begin{array}{l}4.8 \times 1 \times 10^{-3} \\
4.81 \times 10^{-3}\end{array}$ \\
\hline rs12130528 & 1 & 214045514 & $\mathrm{C}$ & 0.03 & $-0.69(0.14)$ & $4.27 \times 10^{-7}$ & $-0.09(0.23)$ & $7.00 \times 10^{-1}$ & $0.29(0.26)$ & $2.50 \times 10^{-1}$ & $-0.32(0.22)$ & $1.5 \times 10^{-1}$ & - & - & $0.28(0.009)$ & $4.69 \times 10^{-3}$ \\
\hline rs 7416880 & 1 & 214038025 & A & 0.03 & $-0.70(0.14)$ & $4.70 \times 10^{-7}$ & $-0.10(0.24)$ & $6.73 \times 10^{-1}$ & $0.30(0.26)$ & $2.47 \times 10^{-1}$ & $-0.33(0.23)$ & $1.5 \times 10^{-1}$ & - & - & $-0.29(0.009)$ & $4.42 \times 10^{-3}$ \\
\hline rs12129576 & 1 & 214038744 & $\mathrm{C}$ & 0.03 & $-0.70(0.14)$ & $4.72 \times 10^{-7}$ & $-0.09(0.24)$ & $6.91 \times 10^{-1}$ & $0.30(0.26)$ & $2.48 \times 10^{-1}$ & $-0.32(0.22)$ & $1.5 \times 10^{-1}$ & -5 & - & $0.28(0.009)$ & $4.88 \times 10^{-3}$ \\
\hline rs12132110 & 1 & 214038803 & A & 0.03 & $-0.70(0.14)$ & $4.77 \times 10^{-7}$ & $-0.09(0.24)$ & $6.93 \times 10^{-1}$ & $0.30(0.26)$ & $2.49 \times 10^{-1}$ & $-0.32(0.22)$ & $1.5 \times 10^{-1}$ & - & - & $-0.28(0.009)$ & $5.05 \times 10^{-3}$ \\
\hline rs11012633 & 10 & 21602727 & A & 0.12 & $0.35(0.07)$ & $8.89 \times 10^{-7}$ & $0.14(0.12)$ & $2.50 \times 10^{-1}$ & $-0.26(0.12)$ & $3.78 \times 10^{-2}$ & $-0.06(0.09)$ & $5.1 \times 10^{-1}$ & - & - & $0.17(0.002)$ & $5.59 \times 10^{-4}$ \\
\hline rs12247067 & 10 & 21603062 & $\mathrm{~T}$ & 0.12 & $0.35(0.07)$ & $8.97 \times 10^{-7}$ & $0.14(0.12)$ & $2.52 \times 10^{-1}$ & $-0.26(0.12)$ & $3.81 \times 10^{-2}$ & $-0.06(0.09)$ & $5.2 \times 10^{-1}$ & - & - & $0.17(0.002)$ & $5.77 \times 10^{-4}$ \\
\hline rs12252084 & 10 & 21607196 & $\mathrm{G}$ & 0.12 & $0.35(0.07)$ & $8.97 \times 10^{-7}$ & $0.14(0.12)$ & $2.53 \times 10^{-1}$ & $-0.23(0.12)$ & $5.98 \times 10^{-2}$ & $-0.06(0.09)$ & $5.2 \times 10^{-1}$ & - & - & $-0.17(0.002)$ & $4.36 \times 10^{4}$ \\
\hline
\end{tabular}


Supplementary Table 3. Human gene expression levels in macular sections of post-mortem donor eyes

All genes represented on the 44k microarray were ranked by increasing expression; rank per gene was calculated as percentile.

Abbreviation: RPE retinal pigment epithelium; SD, standard deviation.

\begin{tabular}{lccc}
\hline Gene & $\begin{array}{c}\text { Photoreceptors } \\
\text { Mean percentile (SD) }\end{array}$ & $\begin{array}{c}\text { RPE } \\
\text { Mean percentile (SD) }\end{array}$ & $\begin{array}{c}\text { Choroid } \\
\text { Mean percentile (SD) }\end{array}$ \\
\hline GOLGA8B & $17(5.0)$ & $14(5.6)$ & $9(3.3)$ \\
GJD2 & $30(2.9)$ & $30(9.3)$ & $36(10.3)$ \\
ACTC1 & $85(8.8)$ & $85(10.2)$ & $89(4.0)$ \\
\hline
\end{tabular}




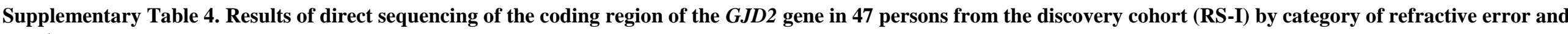
genotype

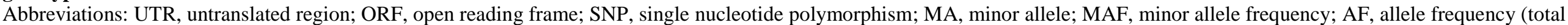
amount of changes (homozygous: single count, heterozygous: double count) per total amount of chromosomes); -, no data.

High myopia: <=-6D, emmetropia: $(-1.5 \mathrm{D}:+1.5 \mathrm{D})$, high hypermetropia $(>=+6 \mathrm{D})$

\begin{tabular}{|c|c|c|c|c|c|c|c|c|c|c|}
\hline \multirow[t]{3}{*}{$\begin{array}{l}\boldsymbol{G J D 2} \\
2 \text { exons, 8 PCR fragments } \\
\end{array}$} & \multicolumn{2}{|r|}{ 5'UTR } & \multicolumn{4}{|c|}{ ORF } & \multicolumn{4}{|c|}{ 3'UTR } \\
\hline & $n$ & SNP1 A->T & SNP2 A->T & SNP3 C->T & SNP4 C->T & SNP5 G->A & SNP6 A->G & SNP7 C->T & SNP8 t->c & SNP9 A->G \\
\hline & & 5UTR-127 & c. $333 \mathrm{~A}->\mathrm{T}$ & c.369 C-> T & c. $.588 \mathrm{C}->\mathrm{T}$ & c. $888 \mathrm{G}->\mathrm{A}$ & $3 \mathrm{UTR}+889$ & $3 \mathrm{UTR}+1069$ & $3 \mathrm{UTR}+1087$ & $3 \mathrm{UTR}+1461$ \\
\hline Amino acid change & & & p.T111T & p.S123S & p.S196S & p.E296E & & & & \\
\hline SNP & & $\begin{array}{c}\text { rs } 2277558 \\
\mathrm{AF} \\
\end{array}$ & $\begin{array}{c}\text { rs651724 } \\
\text { AF }\end{array}$ & $\begin{array}{c}\text { rs35174018 } \\
\text { AF }\end{array}$ & $\begin{array}{c}\text { rs3743123 } \\
\text { AF }\end{array}$ & $\begin{array}{c}\text { rs34964522 } \\
\text { AF }\end{array}$ & $\overline{\mathrm{AF}}$ & $\begin{array}{c}\text { rs607028 } \\
\text { AF }\end{array}$ & $\begin{array}{c}\text { rs792418 } \\
\text { AF }\end{array}$ & $\begin{array}{c}\text { rs2339643 } \\
\text { AF }\end{array}$ \\
\hline \multirow[t]{2}{*}{ High myopia, homozygous for risk allele rs634990 } & 8 & $8 / 16$ & $8 / 16$ & $0 / 16$ & $3 / 16$ & $1 / 16$ & $0 / 16$ & $9 / 16$ & $15 / 16$ & $8 / 16$ \\
\hline & & $(0.500)$ & $(0.500)$ & $(0.000)$ & $(0.188)$ & $(0.063)$ & $(0.000)$ & $(0.562)$ & $(0.938)$ & $(0.500)$ \\
\hline \multirow[t]{2}{*}{ High myopia, homozygous for non risk allele rs634990 } & 8 & $6 / 16$ & $10 / 16$ & $2 / 16$ & $6 / 16$ & $4 / 16$ & $0 / 16$ & $9 / 16$ & $15 / 16$ & $6 / 16$ \\
\hline & & $(0.375)$ & $(0.625)$ & $(0.125)$ & $(0.375)$ & $(0.250)$ & $(0.000)$ & $(0.562)$ & $(0.938)$ & $(0.375)$ \\
\hline \multirow[t]{2}{*}{ Emmetropia, homozygous for risk allele rs634990 } & 8 & $1 / 16$ & $15 / 16$ & $1 / 16$ & $5 / 16$ & $2 / 16$ & $0 / 16$ & $7 / 16$ & $13 / 16$ & $1 / 16$ \\
\hline & & $(0.063)$ & $(0.938)$ & $(0.065)$ & $(0.313)$ & $(0.125)$ & $(0.000)$ & $(0.438)$ & $(0.813)$ & $(0.063)$ \\
\hline \multirow[t]{2}{*}{ Emmetropia, homozygous for non risk allel rs 634990} & 8 & $6 / 16$ & $10 / 16$ & $2 / 16$ & $2 / 16$ & $1 / 16$ & $0 / 16$ & $9 / 16$ & $11 / 16$ & $6 / 16$ \\
\hline & & $(0.375)$ & $(0.625)$ & $(0.125)$ & $(0.125)$ & $(0.063)$ & $(0.000)$ & $(0.563)$ & $(0.688)$ & $(0.375)$ \\
\hline \multirow[t]{2}{*}{ High hypermetropia, homozygous for risk allel rs634990 } & 7 & $4 / 14$ & $10 / 14$ & $0 / 14$ & $5 / 14$ & $3 / 14$ & $0 / 14$ & $8 / 14$ & $13 / 14$ & $4 / 14$ \\
\hline & & $(0.286)$ & $(0.714)$ & $(0.000)$ & $(0.357)$ & $(0.214)$ & $(0.000)$ & $(0.571)$ & $(0.929)$ & $(0.286)$ \\
\hline \multirow[t]{2}{*}{ High hypermetropia, homozygous for non risk allel rs634990 } & 8 & $5 / 16$ & $10 / 16$ & $0 / 16$ & $7 / 16$ & $5 / 16$ & $1 / 16$ & $7 / 16$ & $15 / 16$ & $5 / 16$ \\
\hline & & $(0.313)$ & $(0.625)$ & $(0.000)$ & $(0.438)$ & $(0.313)$ & $(0.063)$ & $(0.438)$ & $(0.938)$ & $(0.313)$ \\
\hline \multirow[t]{2}{*}{ Total } & 47 & $30 / 94$ & $63 / 94$ & $5 / 94$ & $28 / 94$ & $16 / 94$ & $1 / 94$ & $49 / 94$ & $82 / 94$ & $30 / 94$ \\
\hline & & $(0.319)$ & $(0.670)$ & $(0.053)$ & $(0.298)$ & $(0.170)$ & $(0.011)$ & $(0.521)$ & $(0.872)$ & $(0.319)$ \\
\hline MA & & - & $\mathrm{T}$ & A & $\mathrm{T}$ & $\mathrm{T}$ & - & - & - & - \\
\hline MAF (in NCBI SNP database) & & - & 0.267 & 0.059 & 0.267 & 0.053 & - & - & - & - \\
\hline Population (in NCBI SNP database*) & & - & HapMap CEU & AGI_ASP & HapMap CEU & AGI_ASP & - & - & - & - \\
\hline & & & European & population & European & population & & & & \\
\hline$P$-value discovery cohort & & - & $9.45 \times 10^{-1}$ & - & $9.83 \times 10^{-1}$ & - & - & - & - & - \\
\hline MAF discovery cohort & & - & 0.324 & - & 0.282 & - & - & - & - & - \\
\hline
\end{tabular}

*NCBI SNP database http://www.ncbi.nlm.nih.gov/SNP 
Supplementary Table 5. Expression-associated SNPs

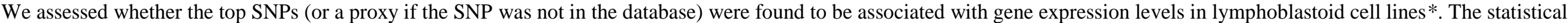
significance threshold was, after applying Bonferroni correction for the number of expression SNPs, defined as a p-value of 3.6x10-3. The $r^{2}$ threshold was defined as 0.70 .

Abbreviations: SNP, single nucleotide polymorphism; -, no data

\begin{tabular}{|c|c|c|c|c|c|c|c|c|c|}
\hline SNP & Proxy & $r^{2}$ & ProbeID & SNP & Allele & Effect & $\begin{array}{c}P \text {-value } \\
\text { Lymphoblastoid cell } \\
\text { line expression }\end{array}$ & $\begin{array}{c}\boldsymbol{P} \text {-value from } \\
\text { GWAS } \\
\text { spherical } \\
\text { equivalent } \\
\text { meta-analysis }\end{array}$ & $\begin{array}{c}\text { Gene } \\
\text { (chromosome location) }\end{array}$ \\
\hline rs11073058 & rs560766 & 0,97 & $1557338 \times$ at & rs560766 & $\mathrm{G}$ & $-0,323$ & $1.0 \times 10^{-5}$ & $7.46 \times 10^{-12}$ & - \\
\hline rs11073059 & rs560766 & 0,97 & 1569714_at & rs560766 & $\mathrm{G}$ & 0,28 & $1.1 \times 10^{-4}$ & $7.46 \times 10^{-12}$ & ZFYVE20 (3p25.1) \\
\hline rs11073060 & rs560766 & 0,97 & 1557338_x_at & rs580839 & $\mathrm{C}$ & $-0,307$ & $9.5 \times 10^{-6}$ & $2.53 \times 10^{-11}$ & - \\
\hline rs4924134 & rs560766 & 0,97 & 227941_at & rs580839 & $\mathrm{C}$ & $-0,268$ & $1.4 \times 10^{-4}$ & $2.53 \times 10^{-11}$ & LOC339803 (2p15) \\
\hline rs524952 & rs560766 & 0,71 & AVG_TBC1D1 & rs580839 & $\mathrm{C}$ & $-0,266$ & $1.9 \times 10^{-4}$ & $2.53 \times 10^{-11}$ & - \\
\hline rs560766 & - & - & 227903 _x_at & rs811563 & A & 0,356 & $6.8 \times 10^{-6}$ & $6.0 \times 10^{-1}$ & C19orf20 (19p13.3) \\
\hline rs580839 & - & - & 1563985_at & rs811563 & A & $-0,34$ & $1.4 \times 10^{-5}$ & $6.0 \times 10^{-1}$ & - \\
\hline rs619788 & rs580839 & 1 & AVG_ZNF653 & rs811563 & $\mathrm{A}$ & 0,331 & $2.0 \times 10^{-5}$ & $6.0 \times 10^{-1}$ & - \\
\hline rs634990 & rs560766 & 0,68 & 223371 s at & rs811563 & A & 0,337 & $2.3 \times 10^{-5}$ & $6.0 \times 10^{-1}$ & DNAJC4 (11q13) \\
\hline rs685352 & rs 811563 & 0,6 & 220077_at & rs811563 & $\mathrm{A}$ & 0,316 & $5.6 \times 10^{-5}$ & $6.0 \times 10^{-1}$ & FLJ22349 (22q13.2) \\
\hline rs688220 & rs580839 & 0,97 & 1558212 at & rs811563 & A & $-0,314$ & $6.5 \times 10^{-5}$ & $6.0 \times 10^{-1}$ & FLJ35024 (9p24.2) \\
\hline rs7163001 & rs560766 & 0,97 & 1560199_x_at & rs811563 & A & $-0,321$ & $6.6 \times 10^{-5}$ & $6.0 \times 10^{-1}$ & $\cos ^{-60}$ \\
\hline rs7176510 & rs560766 & 0,9 & 234020_x_at & rs811563 & A & $-0,314$ & $7.1 \times 10^{-5}$ & $6.0 \times 10^{-1}$ & COMMD1 (2p15) \\
\hline \multirow[t]{9}{*}{ rs8032019 } & rs560766 & 0,78 & AVG HIVEP3 & rs811563 & $\mathrm{A}$ & $-0,314$ & $8.7 \times 10^{-5}$ & $6.0 \times 10^{-1}$ & - \\
\hline & & & 212349_at & rs811563 & $\mathrm{A}$ & 0,314 & $9.5 \times 10^{-5}$ & $6.0 \times 10^{-1}$ & POFUT1(20q11) \\
\hline & & & 241602 at & rs811563 & $\mathrm{A}$ & $-0,31$ & $1.1 \times 10^{-4}$ & $6.0 \times 10^{-1}$ & ZNF582 (19q13.43) \\
\hline & & & 237766_at & rs811563 & $\mathrm{A}$ & 0,298 & $1.2 \times 10^{-4}$ & $6.0 \times 10^{-1}$ & ATP9B (18q23) \\
\hline & & & 206468_s_at & rs811563 & A & 0,305 & $1.6 \times 10^{-4}$ & $6.0 \times 10^{-1}$ & METTL13 (1q24-q25.3) \\
\hline & & & 244092_at & rs811563 & A & $-0,293$ & $1.7 \times 10^{-4}$ & $6.0 \times 10^{-1}$ & ZRANB3(2q21.3) \\
\hline & & & 207660 at & rs811563 & $\mathrm{A}$ & $-0,293$ & $1.7 \times 10^{-4}$ & $6.0 \times 10^{-1}$ & $D M D(\mathrm{Xp} 21.2)$ \\
\hline & & & AVG_ALCAM & rs811563 & A & $-0,293$ & $1.8 \times 10^{-4}$ & $6.0 \times 10^{-1}$ & $\begin{array}{c}- \\
-\end{array}$ \\
\hline & & & 227098_at & rs811563 & $\mathrm{A}$ & $-0,298$ & $1.9 \times 10^{-4}$ & $6.0 \times 10^{-1}$ & DUSP18 (22q12.2) \\
\hline
\end{tabular}

*Dixon, A.L. et al. A genome-wide association study of global gene expression. Nat Genet 39, 1202-7 (2007). 
Supplementary Figure 1. Quantile-quantile plots for discovery and replication studies

SNPs had minor allele frequency $>=0.01$. Each black circle represents an observed statistic (defined as $\log _{10} P$ ) versus the corresponding expected statistic. The red line corresponds to the null distribution. Values were adjusted by genomic controls within each study ( $\lambda=1.054$ for RS-I, 1.012 RS-II, 1.012 RS-III, 1.037 for ERF and 1.04 for TwinsUK).

Discovery cohort RS-I

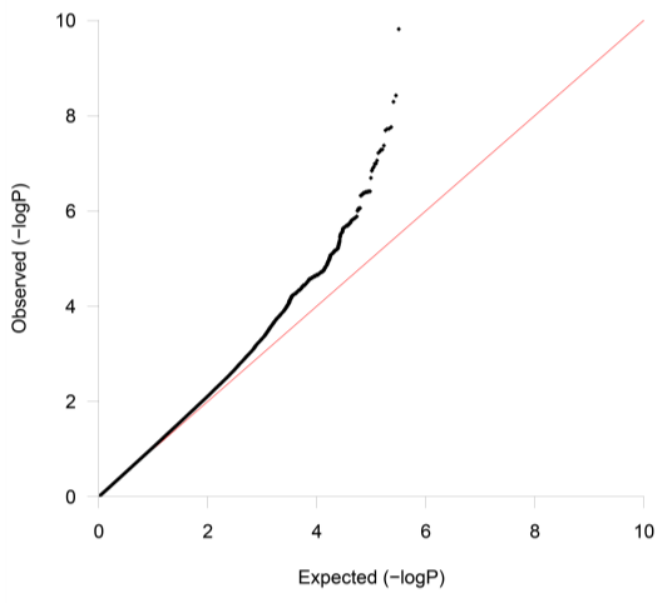

RS-III

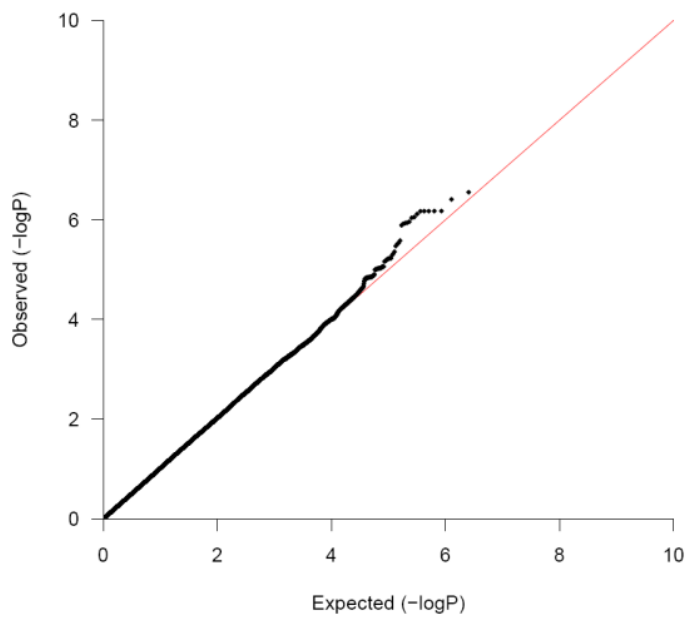

RS-II

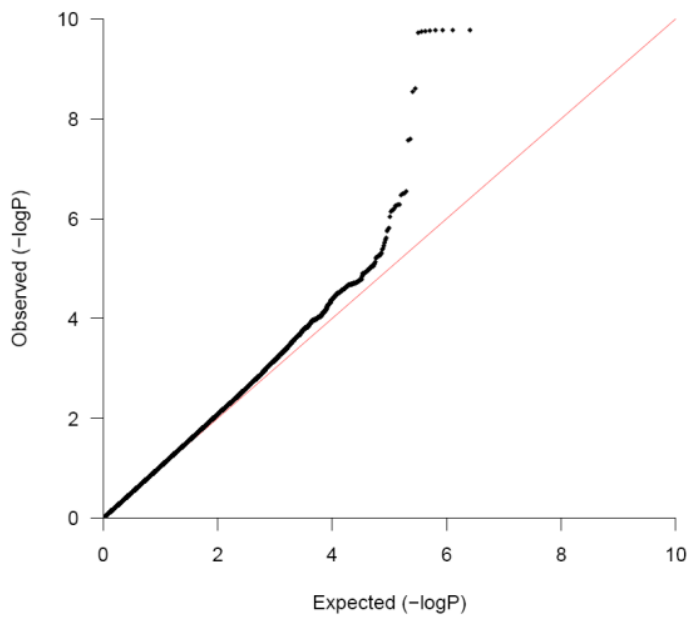

ERF

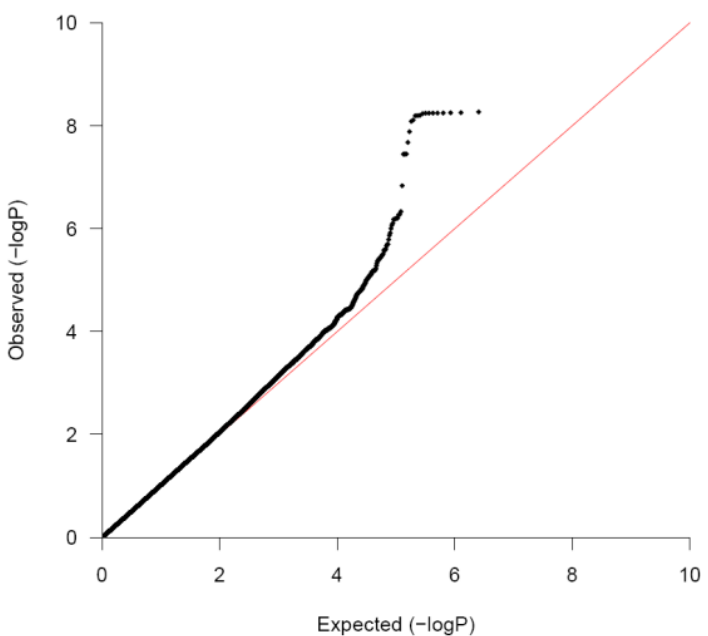

TwinsUK

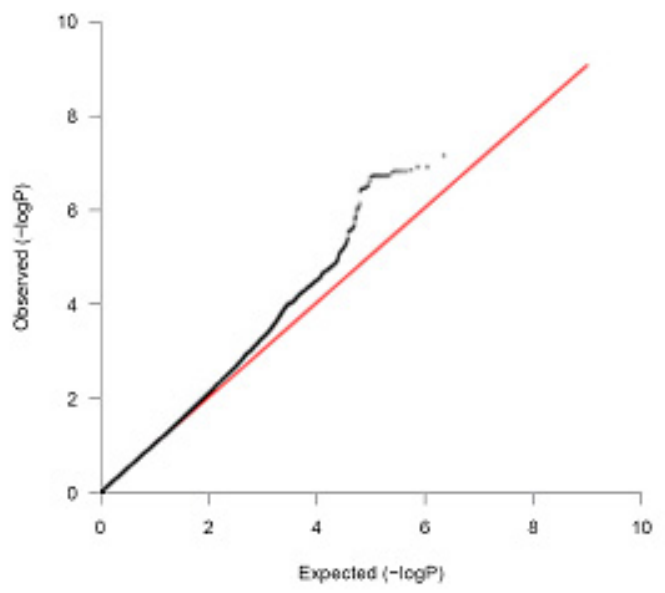




\section{Supplementary Figure 2. Ingenuity analysis of the GJD2 gene}

\section{Supplementary Figure 2a. GJD2 and the interaction with eye development genes}

Exploration of the Ingenuity database provided a large number of functional links between REST (RE1-silencing transcription factor), insulin, SP1 (Sp1 transcription factor), CAV1 (caveolin 1)). For example, direct proteinprotein interaction was found with CAV1, a plasma membrane involved in cellular growth, apoptosis, migration and cell survival during eye development. Interestingly, transcription of GJD2 is, via SP1, apparently under similar control as growth factors such as VEGFA (vascular endothelial growth factor A) and EGFR (epidermal growth factor receptor), which are also known to be involved in eye growth and development. This analysis suggests that GJD2 is expressed during eye development and that the protein plays a functional role in this process.

\section{Supplementary Figure 2b. GJD2 interactions with other membrane-bound and connexin proteins} This search yielded four genes (GJA8 (gap junction protein, alpha 8), VEGFA, FGF2 (fibroblast growth factor 2), RLBP1(retinaldehyde binding protein 1)). GJA8 is a protein family member of GJD2 (CX36). We observed a functional molecular network via direct protein-protein interactions, connecting, the gap junction proteins GJA1 (gap junction protein, alpha 1), GJA3 (gap junction protein, alpha 3), GJA8 and GJD2, together with TJP1 (tight junction protein 1) and CAV1. Transgenic mouse and in vitro models previously showed that two of the GJD2 family members (GJA3, GJA8) are involved in eye morphogenesis, determination of eye size, or lens fiber maturation****. Since this network suggests that all molecules shown act in the same or similar molecular complex involved in eye growth and lens fiber maturation, it suggests that GJD2 is involved in both these mechanisms.

*Rong P. et al. Disruption of Gja8 ( $\alpha 8$ connexin) in mice leads to microphthalmia associated with retardation of lens growth and lens fiber maturation. Development 129, 167-174 (2002).

**White, T.W. et al. Targeted Ablation of Connexin50 in Mice Results in microphthalmia and Zonular Pulverulent Cataracts. J Cell Biol 143, 815-825 (1998).

\section{Legend}

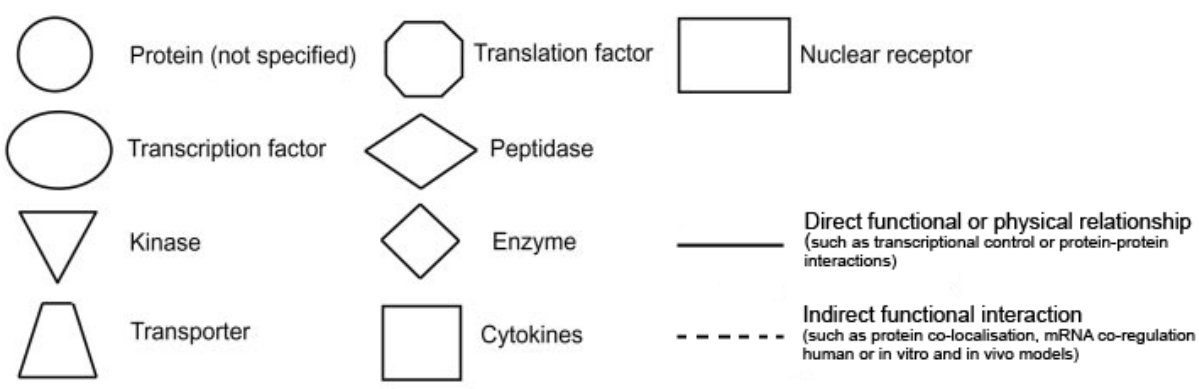




\section{Supplementary Figure 2a.}

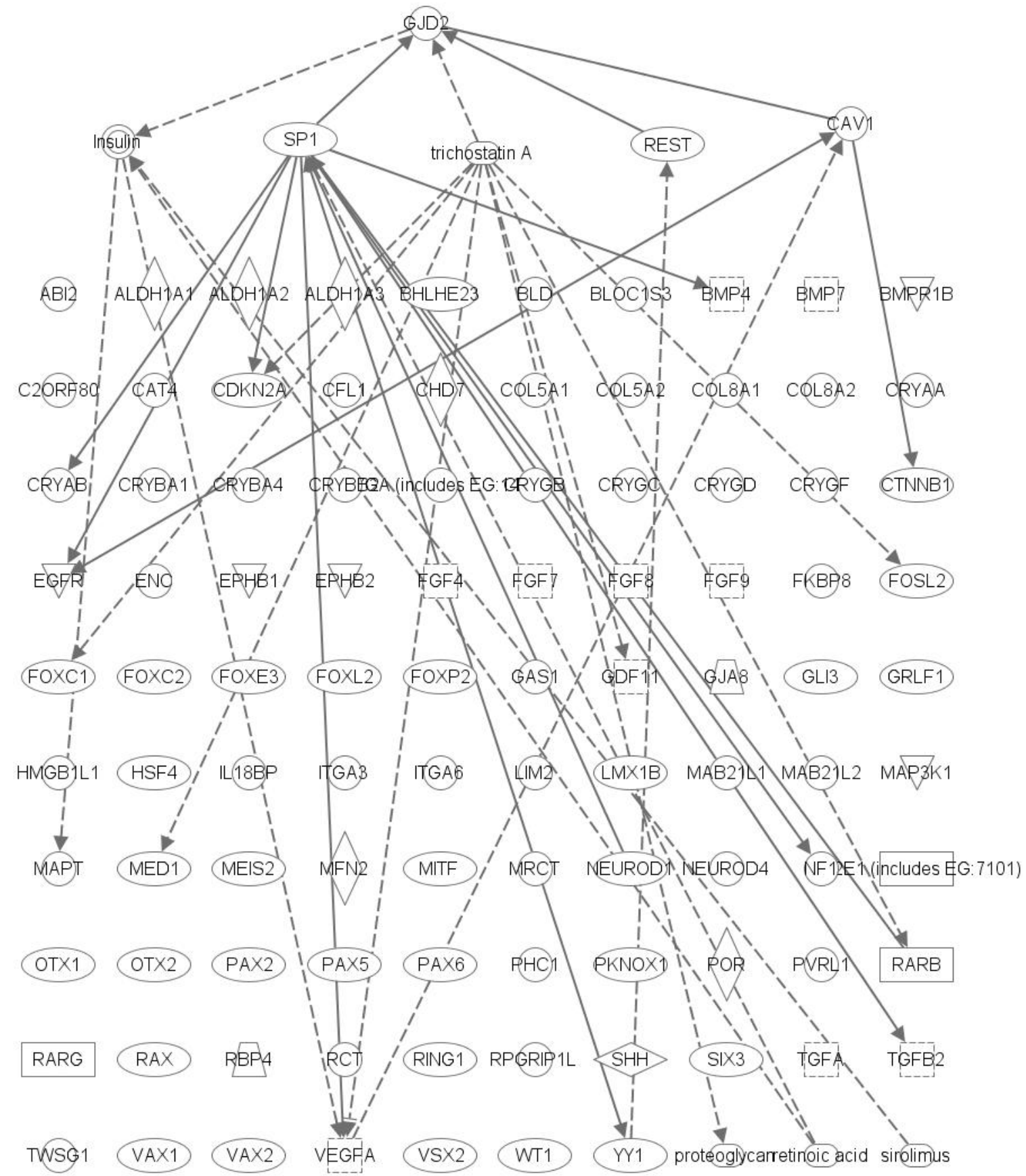

\section{Supplementary Figure 2b.}

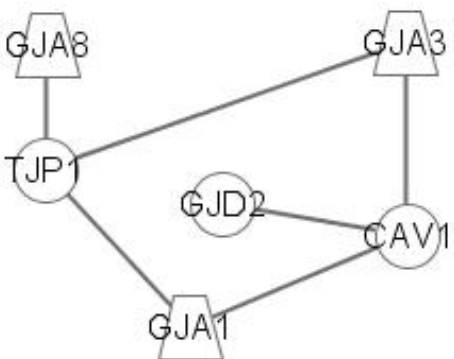

(C)2000-2010 Ingenuity Systems, Inc. All rights reserved. 
Supplementary Figure 3. Regulatory elements on the $15 q 14$ associated locus

Regional map of the associated locus based on UCSC Genome browser views (Mar. 2006 (hg 18), NCBI Build 36.1, chr15:32766300-32820148 bp, and May 2004 (hg17),

NCBI Build 35, chr15:32766300-32820148 bp) with used borders SNP nr rs7494782-rs644339 with accompanying DNase I hypersensitive sites (represented by the grey

blocks), sequence conservation (represented by the blue peaks) and the insulator site in terms of CTCF binding (illustrated in light blue)*.

*Heintzman, N.D. \& Ren, B. Finding distal regulatory elements in the human genome. Curr Opin Genet Dev 19, 541-9 (2009).

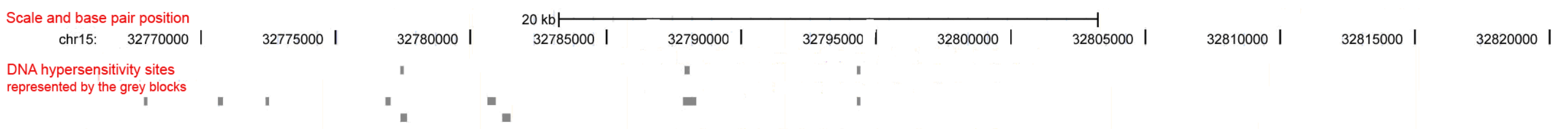

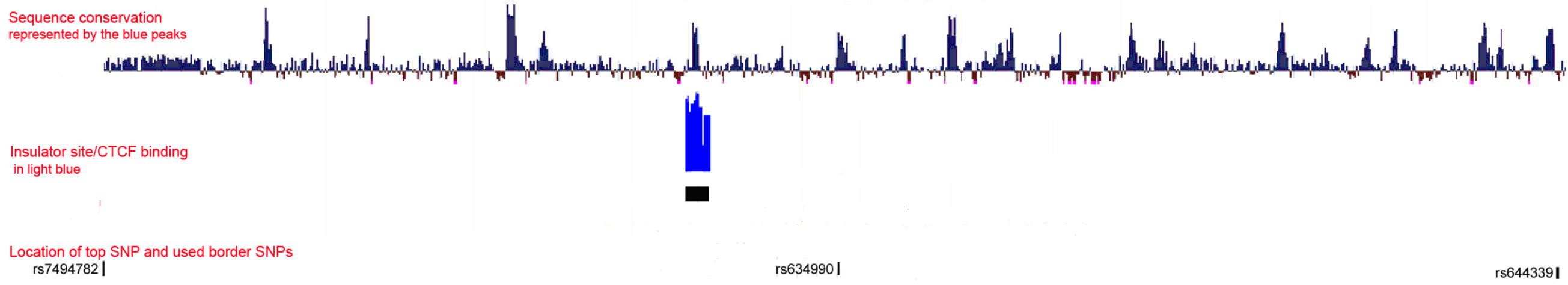

